\title{
A General Actuator Saturation Compensator in the Continuous-Time Domain
}

\author{
C. W. Chan and K. Hui \\ Department of Mechanical Engineering \\ University of Hong Kong, Pokfulam Road, Hong Kong
}

\begin{abstract}
Abstruct - A general compensator for actuator saturation that includes existing ones as special cases is presented. The conditions that must be satisfied for the implementation of the compensator are given. It is shown that for a given system there exists an arbitrarily large number of compensators such that the compensated system is absolute stable. The resulf suggests that a compensator can be derived from systems that is known to be absolute stable. If the system is unknown, then the compensator may have to be designed iteratively until the effective set-point is acceptable.
\end{abstract}

\section{INTRODUCTION}

Actuator saturation is a common problem in practical control systems, and if it is not being compensated properly, the closed-loop system can become unstable, In earlier works [1], anti-reset windup schemes are devised specifically to unwind the integrator of controllers such as the PI or PID controllers. With the advent of microprocessors, more general compensation schemes $[2,3]$ are devised. The algorithms are derived in discrete time and are generally unsuitable for implementation in continuous time. Further, these techniques are derived on an ad hoc basis, the effect on the performance and the stability of the closed-loop systems are little known.

A general actuator saturation compensator which can be implemented in both continuous and discrete time is presented. The main contribution of this formulation is that it provides a framework to study the design of the compensator and the stability of the compensated system. Also, it is shown that some of the existing schemes [5,4] are included as special cases. The motivation for the new formulation is as follow. When the actuator saturates, the control cannot be fully implemented by the actuator. The difference between the unconstrained control and the actuator limit, denoted by $\delta$, can be considered as a disturbance being injected into the closed-loop system. Through suitable transformation, the set-point is now altered by the transformed disturbance, giving the effective set-point.
The alternation of the set-point occurs whenever the actuator saturates, irrespective whether a compensator is used. Using a particular compensator merely specifies the transformation of the set-point. A useful outcome of this interpretation of actuator saturation is a general formulation of its compensation. Compensation for actuator saturation can now be implemented by adding a term involving $\delta$ in the controller. The conditions that must be satisfied for the implementation of compensators using this formulation are discussed. Also, it is shown that there exists an arbitrarily large number of compensators such that the compensated system remains stable irrespective of the actuator limits. The result is useful for designing a compensator for a system with known transfer function. If the transfer function is unknown, then the compensator may have to be chosen iteratively until the effective set-point is acceptable.

\section{A GENERAL COMPENSATION SCHEME}

Let $G(s)$ be a rational transfer function. The controller, in the input-output form, is given below.

$R(s) \mathbf{v}(s)=T(s) w(s)-S(s) y(s)$,

where $y(s), w(s)$ and $v(s)$ are respectively the output, the set-point and the control; $R(s), T(s)$ and $S(s)$ are rational functions in $s$. The leading coefficients of both the numerator and the denominator polynomials of $\mathbf{R}$ are assumed to be unity. The root of a polynomial is said to be stable if the real part of the root is non-positive, and asymptotically stable if the real part is negative real. The closed-loop system satisfies the following assumptions.

A1 The poles of $\mathrm{G}(\mathrm{s})$ are stable.

A2 The linear closed-loop system is asymptotically stable, i.e., the zeros of $(R+G S)$ are asymptotically stable.

A3 The controller is stable, i.e., the zeros of $R$, and the poles of $T$ and $S$ are stable.

Let $u(t)$ be the output of the actuator given by 


$$
u(t)=\left\{\begin{array}{rr}
u_{\max } & v(t)>u_{\max } \\
v(t) & u_{\min } \leq v(t) \leq u_{\max } \\
u_{\min } & v(t)<u_{\operatorname{mix}}
\end{array}\right.
$$

where $u_{\max }$ and $u_{\min }$ are the upper and lower limits of the actuator. When the actuator saturates. $v(t)$ cannot be fully implemented. The control that cannot be implemented can be interpreted as a non-linear disturbance $\delta(t)$. computed posteriori as shown below.

$$
\delta(t)=u(t)-v(t)=\left\{\begin{array}{lr}
u_{\max }-v(t) & v(t)>u_{\max } \\
0 & u_{-} \leq v(t) \leq u_{\max } \\
u_{\min }-v(t) & v(t)<u_{m}
\end{array}\right.
$$

Equation (1) can be modified as follows to include the effect of actuator saturation and its compensation [6].

$$
R v=T w-S y+P \delta
$$

where $P$ is a rational function in $s$. The argument $s$ has been dropped for convenience. For discrete implementation, simply replaces $\mathrm{s}$ by $\mathrm{z}^{-1}$ for the rational functions and by $t$ for the variables. Choosing a compensator now reduces to choosing $P$. If $P=0$, then the control is computed ignoring actuator saturation. Other choices of $P$ are given in Section $V$. The choice of $P$ is subjected to two conditions. The first is that it is realizable and the second. the compensated system remains stable. Dividing both sides of (4) by $R$, then the compensation scheme is realizable if the following condition is satisfied.

\section{A4 $\mathrm{P}(\mathrm{s}) / \mathrm{R}(\mathrm{s})<\infty$, as $\mathrm{s} \rightarrow \infty$}

The block diagram of the compensated closedloop system is shown in Fig. 1. The output of the compensated system is

$$
y=\frac{G T}{R+G S} w+\frac{G(R+P)}{R+G S} \delta=\frac{G T}{R+G S} \bar{w},
$$

where $\bar{w}$ is the effective set-point given by

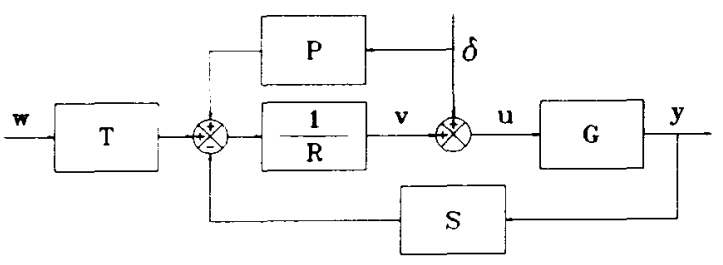

Fig.1 Closed-loop system with actuator saturation compensation

$$
\bar{w}=w+\frac{R+P}{T} \delta
$$

From (5), there is no actuator saturation if $w$ is replaced by $\vec{w}[2]$. The stability of the compensated system is determined by the term associated with $\delta$ once the actuator saturates since the linear closed-loop system is asymptotically stable by $A 2$. The poles of this term are given by the zeros of $(R+G S)$ and the poles of $P$. The former is asymptotically stable from $A 2$, hence it is necessary for the poles of $P$ to be stable to ensure the stability of this term. Rearranging (5), $\delta$ can be written as

$$
\delta=\frac{1}{R+P}[(R+G S) u-T w]
$$

Clearly, $\delta$ is asymptotically stable if and only if both the zeros of $(R+P)$ and the term associated with $\mathrm{u}$ are asymptotically stable. Hence, $(\mathrm{R}+\mathrm{P})$ having asymptotically stable poles is a necessary condition for the compensated system to be asymptotically stable. These conditions are summarized in A5.

A5 The zeros of $(R+P)$ and the poles of $P$ are asymptotically stable.

The result discussed so far is all that can be obtained using linear analysis. Further results on the stability of the term involving $u$, a non-linear function, can only by obtained using non-linear analysi's [7], as discussed in Section III. If A5 is not satisfied, the compensated system may still be asymptotically stable for certain actuator limits, but not for all actuator limits.

The stability of the effective set-point $\bar{w}$ depends not only on conditions given in A5, but also on the stability of the zeros of $T$. If $T$ contains unstable zeros that have not been cancelled by that of $(R+P), \bar{w}$ 
becomes unstable, though the compensated system is stable. It is mainly for this reason that compensation schemes are usually implemented using (4) instead of using directly the effective set-point.

\section{STABILITY OF THE COMPENSATED SYSTEMS}

The block diagram shown in Fig. 1 is first reduced to a standard form shown in Fig. 2. The modified block diagram consists of a linear block, $G_{\mathrm{E}}$ and a non-linear block $f(v)$, representing the actuator saturation defined below.

Definition D1 The non-linearity $f(v)$ is a real, continuous, single-valued, scalar function of the real scalar argument $\mathrm{v}$, satisfying the following conditions,

$$
f(v)=0, \text { for } v=0
$$

If $\mathrm{f}(\mathrm{v}) / \mathrm{v}=1$, there is no actuator saturation; and the smaller is the ratio, the more severe is the saturation. To derive $T_{E}$ and $G_{E}$, first rearrange (3),

$\delta=\mathrm{u}-\mathrm{f}(\mathrm{v})$

Substituting (8) into (4), and rearranging gives

$$
v=\frac{T}{R+P} w-\frac{G S-P}{R+P} f(v)
$$

from which,

$$
T_{E}=\frac{T}{R+P}, \quad G_{E}=\frac{G S-P}{R+P} .
$$

The term $T_{E}$ can be ignored in stability analysis, since $w$ is often set to zero and the term involving $w$ is asymptotically stable if A3 and A5 are assumed. In the following, a system is said to be absolute stable if it is asymptotically stable irrespective of the actuator limits.

Theorem 1 For a closed-loop system satisfying A1 to $A 3$, with a non-linearity $f(v)$ given by $D 1$, there exists an arbitrarily large number of compensation schemes given by (4) with P satisfying A4 and A5 such that the compensated system is absolute stable.

Proof Let $\overline{\mathrm{G}}_{\mathrm{E}}$ be a rational function such that the non-

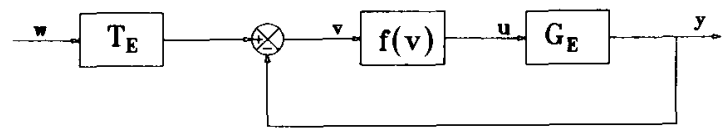

Fig.2 Simplified block diagram

linear closed-loop system is absolute stable. From (10), $P$ is uniquely given by

$P=\frac{G S-G_{E} R}{1+G_{E}}$.

It is shown in the following that P satisfies A4 and A5. The poles of $P$ is given by the zeros of $\left(1+\bar{G}_{E}\right)$ and the poles of $G, S$ and $R$, all of which are stable since $A 1$ to $A 3$ are assumed. From (11), the zeros of $(R+P)$ is the same as that of $(R+G S)$ which are asymptotically stable from A2. Hence A5 is satisfied. Dividing (11) by $R$ gives,

$\frac{P}{R}=\frac{\bar{G}-\bar{G}_{E}}{1+\bar{G}_{E}}$,

where $\mathbf{G}=\frac{\mathrm{GS}}{\mathrm{R}}$,

Since $\bar{G}_{E}, G$ and $S / R$ are proper by assumption, hence $P / R$ given in (12) is also_proper. There exists an arbitrarily large number of $G_{E}$, and from (12), so is $P$.

Remark 1 The result given in Theorem 1 is general and applies to other non-linearity such as deadband.

Remark 2 Although the compensated system may be absolute stable, the transient response of the compensated system may not always be acceptable.

Absolute stability is a stringent requirement for practical systems since actuator are often designed with spare capacity over the steady-state requirement. For this reason, compensator designed for stability over certain range of actuator saturation is of more practical interest.

\section{COMPENSATOR DESIGN}


There are two approaches to design a compensator. If $G(s)$ is available, then $\underline{P}$ can be computed from (11) by first specifying $\bar{G}_{E}$ which satisfies the conditions for absolute stable. If a design criterion less stringent than absolute stability is adopted, then $P$ can be chosen by specifying the phase margin of the compensated system, i.e., $\bar{G}_{\mathrm{E}}$. In industrial applications where $G(s)$ is not available, $P$ can be chosen iteratively using the effective set-point as a guideline. Equation (6) can be rewritten as

$\overline{\mathbf{w}}=\mathbf{w}+\Gamma \delta$

where

$\Gamma=\frac{\mathrm{R}+\mathrm{P}}{\mathrm{T}}$.

$P$ can be expressed in terms of $\Gamma$ as follow.

$P=T \Gamma-R$

If $\Gamma$ is chosen such that the poles of $T \Gamma$ are stable and that $P$ satisfies $A 4$ and $A 5$, then a compensator can be chosen by specifying $\Gamma$. Note that, from (6), specifying $\Gamma$ only determines partially $\bar{w}$, since $\delta$ is a non-linear function given by (3). Consequently, designing a compensator using this approach would naturally be iterative.

\section{EXISTING SCHEMES}

A survey of actuator saturation compensation schemes can be found in [5]. The common ones are [4]: (i) the use controller output (UCO) scheme that ignores actuator saturation in computing the control, (ii) the use actuator output (UAO) scheme that uses the actuator output to update the control and (iii) the instantaneous set-point adjustment (ISA) that uses the adjusted set-point to update the control. The adjusted set-point is computed posteriori such that if the adjusted set-point is used, the actuator does not saturate. The choice of $P$ for these schemes is shown in Table I. Note that in Table $I, t_{0}=T(s) / s^{n}$, as $s \rightarrow \infty$, where $n$ is the larger of the degrees of the numerator and the denominator polynomials of $\mathrm{T}$.

\section{EXAMPLES}

TABLE I

P FOR EXISTING SCHEMES

\begin{tabular}{l|ccc} 
& UCO & UAO & ISA \\
\hline$P$ & 0 & $1-R$ & T/t。 $-\mathrm{R}$
\end{tabular}

Two examples simulated on an analogue computer are presented here. The first illustrates the implementation of the compensators discussed previously, and the second, on the design of a compensator for absolute stability.

Example 1 Let $\mathrm{G}(\mathrm{s})=\omega_{0}{ }^{2} / \mathrm{s}^{2}$, a double integrator. The controller is given by

$v(s)=\left(K+\frac{1}{T_{i} s}\right)(w(s)-y(s))-T_{d} s y-(s)$.

Let $\omega_{0}=1, K=1, T_{i}=4$ and $T_{d}=2$, then $P$ for the schemes described previously are shown in Table II. Clearly, P satisfies A4 and A5. For actuator limits of \pm 0.05 , the output of the compensated system using respectively the UAO and the ISA schemes for a step input of 5 units are shown in Fig. 3. The output using the UCO scheme has not been shown since the compensated system is unstable. Clearly, using the UAO scheme yields the best result. It should be noted that since none of the schemes is an optimal design, it is not expected using the UAO scheme, or any other schemes would always yield the best result.

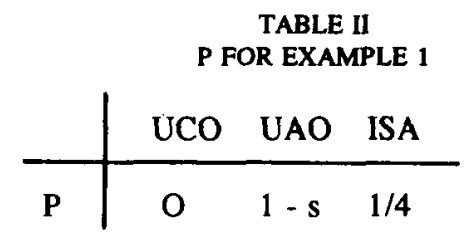

Example 2 Let $G(s)=1 /(s+0.1)^{2}, R(s)=s^{3}+5.2 s^{2}$ $+7.25 \mathrm{~s}, \mathrm{~S}(\mathrm{~s})=11.25 \mathrm{~s}^{2}+4.74 \mathrm{~s}+3.0125$ and $\mathrm{T}(\mathrm{s})=$ $2.41(s+0.2)(s+2.5)^{2}$. For the ISA scheme, $P=1.25$, and

$G_{E}(s)=\frac{10\left(s^{2}+0.45 s+0.30\right)}{(s+0.1)^{2}(s+0.2)(s+2.5)^{2}}$

The output of the compensated system is shown in Fig. 4a for a step input of 5 and actuator limits of \pm 0.1 . A 


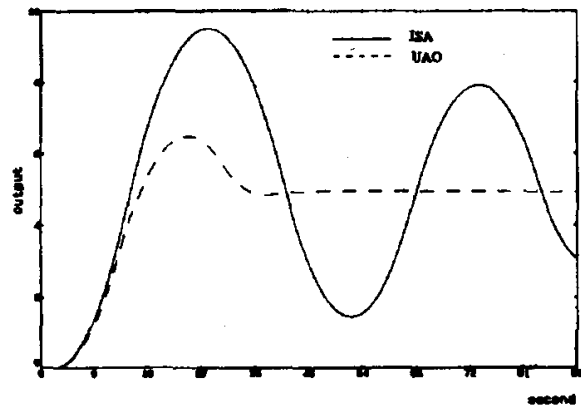

Fig. 3 Closed-loop output using respectively ISA and UAO schemes

stable limit cycle is observed. If $P$ is chosen such that $\mathrm{G}_{\mathrm{E}}(\mathrm{s})=10 /(\mathrm{s}+0.1)$, then

$P(s)=\frac{-10 . s^{4}-53 s^{3}-66.45 s^{2}-2.5 s+3.0125}{(s+0.1)(s+10.1)}$

The output of the compensated system is shown in Fig. 4b. There is no limit cycles and the output reaches the set-point quickly. Though better performance is obtained, implementing the compensator is more complex than that for the ISA scheme.

\section{CONCLUSIONS}

A general actuator saturation compensator that includes existing compensators as special cases is presented. The existence of an arbitrarily large number of compensators such that the compensated system is absolute stable is also established. The compensator design is discussed and the results illustrated with examples simulated on an analogue computer.

\section{ACKNOWLEDGMENT}

The authors wish to thank C. L. Ting for preparing the simulation results.

\section{REFERENCE}

[1] Fertik, H. A. and C. W. Ross, "Direct digital control algorithm with anti-windup feature", ISA Trans., vol. 6, no. 4, 1967, pp. 317-328. Hanus, R., "A new technique for preventing control windup", Journal A, vol. 21, no. 1, 1980, pp.15-20.
[3] Walgama, K. S., S. Rönnbäck and J. Sternby, "Generalisation of conditioning technique for anti-windup compensators", Proc. IEE Pt.D, vol. 139, no. 2 , 1992 , pp. 109-118.

Chan C. W. and K. Hui, "Actuator saturation compensation for self-tuning controllers", Int. $J$. Control, vol. 59, no. 2, 1994, 543-560.

[5] Hanus, R, "Antiwindup and bumpless transfer: a survey", Computing and Computers for Control Systems, edited by P. Borne et al., J. C. Baltzer AG, Amsterdam, The Netherlands, 1989 , p. 3-9.

[6] Hui, K and C. W. Chan, "Stability analysis of actuator saturation compensation schemes", Proc. CAI Symposium '94, HKIE, 1994, pp. 1525.

[7] Narendra, K. S. and J. H. Taylor, Frequency Domain Criteria for Absolute Stability, Academic Press, 1973.

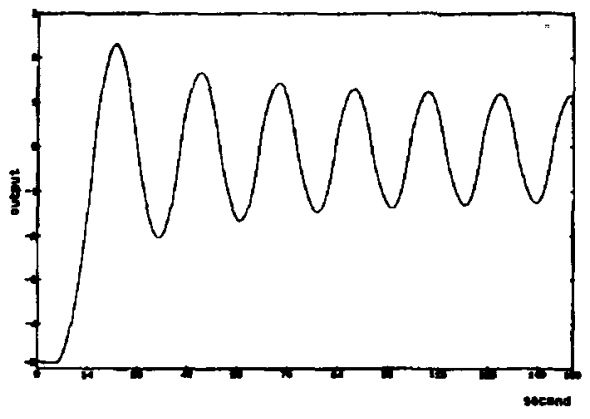

Pig. 4a Closed-loop output using ISA scheme

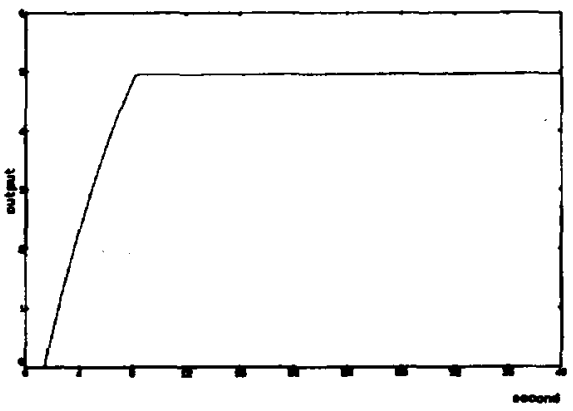

Fig. 4b Closed-loop output using $P$ given by (17) 